\title{
Provider satisfaction with an inpatient tobacco treatment program: results from an inpatient provider survey
}

This article was published in the following Dove Press journal:

International Journal of General Medicine

19 October 2017

Number of times this article has been viewed

\section{Susan Trout \\ Carol Ripley-Moffitt \\ Clare Meernik \\ Jennifer Greyber \\ Adam O Goldstein}

Nicotine Dependence Program, Department of Family Medicine, University of North Carolina School of Medicine, Chapel Hill, NC, USA

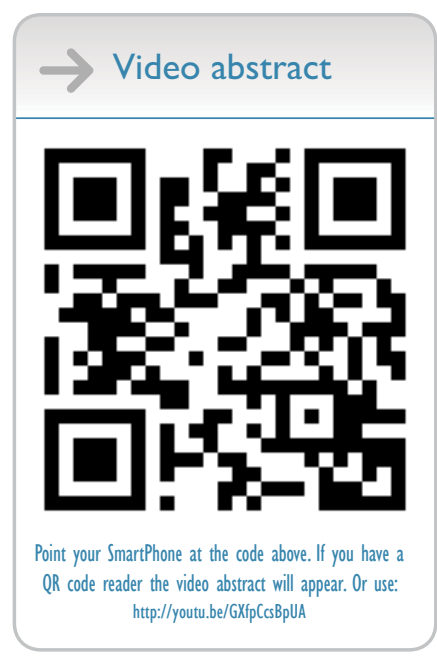

Correspondence: Susan Trout Department of Family Medicine, University of North Carolina School of Medicine, 590 Manning Drive, CB \#7595, Chapel Hill, NC 27599, USA

Tel +l 9849748453

Fax + I 9849748606

Email susan_trout@med.unc.edu
Background: Hospitalization offers an optimal environment for ensuring that patients receive evidence-based treatment. An inpatient tobacco treatment program can deliver interventions broadly, but minimal research has examined the impacts of a consult program on inpatient providers. The Nicotine Dependence Program at the University of North Carolina has provided an inpatient tobacco treatment consult service since 2010.

Objective: The program sought feedback from inpatient providers to examine factors that prompted tobacco treatment consult orders, the impact on provider counseling behavior, provider satisfaction, and suggested program improvements.

Design: Providers who had ordered a tobacco treatment consult received an online anonymous survey.

Setting: The University of North Carolina Hospital is an academic medical facility with 803 beds and over 37,000 inpatient admissions annually from all 100 counties in North Carolina. Approximately $20 \%$ of these inpatients report current use of any tobacco product.

Patients/participants: Medical providers who ordered inpatient tobacco treatment consults from July 2012 to June 2013 ( $\mathrm{n}=265$ ) received the survey, with 118 providers responding $(44.5 \%$ response rate).

Results: Almost all providers reported being satisfied with the consult program and believed it was effective. Key factors in provider satisfaction included ease of accessing the service, saving provider time, and offering patients evidence-based tobacco use treatment. The consult program increased the likelihood of providers prescribing tobacco cessation medications at discharge, as well as following up at post-discharge appointments.

Conclusion: This is some of the first research to show provider satisfaction, program usage, and outcomes with an inpatient tobacco treatment program and demonstrates the important impact of implementing tobacco treatment services within hospitals.

Keywords: provider satisfaction, tobacco, tobacco treatment counseling, inpatient, hospital, smoking, smoking cessation

\section{Introduction}

National guidelines recommend that health care networks support patients who want to quit tobacco use by developing systems that ensure consistent identification of tobacco use status and offering effective tobacco use treatment that incorporates both pharmacotherapy and ongoing behavioral counseling to every person who uses tobacco. ${ }^{1}$ Opportunities exist to dramatically increase the reach and effectiveness of tobacco treatment services by integrating educational, systems-based, and quality improvement strategies for inpatient tobacco use treatment interventions linked with ongoing outpatient counseling and medication. ${ }^{2}$ 
Hospitalization offers an optimal environment for identifying tobacco use and ensuring that patients receive evidencebased treatment. ${ }^{2-4}$ Some studies suggest that hospitalized patients are primed to participate in health education activities during times when they are not actively involved in other health care treatments. ${ }^{5}$ Research also shows that even a brief cessation intervention in a clinical setting can help tobacco users quit. ${ }^{6}$ Additionally, comprehensive tobacco-free policies at an increasing number of hospitals require patient abstinence, and illness provides a teachable moment when patients are more likely to be open to behavior change. ${ }^{2,7-9}$ Hospital settings allow for involvement of multiple providers, ease of access to tobacco cessation medications, and the opportunity for patient counseling, while negating time and travel as barriers for participation. ${ }^{10}$ Recent literature reviews conclude that programs combining inpatient counseling with post-discharge support for a minimum of 4 weeks increase a patient's chances of quitting by $65 \% .{ }^{11}$ In light of these findings, the Joint Commission has approved four measures related to inpatient tobacco use: screening, treatment during hospitalization, treatment management/referral at discharge, and assessing status after discharge. ${ }^{12}$ These voluntary tobacco measures are one of the six core measures that can be used for hospital accreditation. ${ }^{12}$ While there are some published studies that describe models for integrating such measures into hospital systems, ${ }^{13-16}$ to the best of our knowledge, this is the first study that describes the impact of such programs on provider knowledge and practice related to inpatient tobacco use treatment.

\section{Study context}

Our institution's health care system operates an 803-bed teaching hospital with an average daily admission of 100 patients, $\sim 20 \%$ of whom indicate current tobacco use, which includes smoked and smokeless products. In 2010, the Nicotine Dependence Program (NDP), our medical center's tobacco treatment program, developed and implemented the Inpatient to Outpatient program, a formal tobacco treatment consult service for inpatients at University of North Carolina (UNC) Hospital in Chapel Hill. The referral-based service utilizes the hospital's electronic health record order sets, which allow providers to request a bedside consult from a tobacco treatment specialist and to prescribe tobacco cessation medications. Our consult team of tobacco treatment specialists provides bedside assessment and counseling, recommends tobacco cessation medications when appropriate, and makes post-discharge recommendations and referrals. In order to better assess success at dissemination of inpatient tobacco use treatment and its overall impact on provider behavior, the NDP conducted a survey with providers who had utilized the inpatient tobacco treatment consult service between July 2012 and June 2013.

\section{Subjects and methods Survey development}

We designed the survey based on aspects of the consult service about which we desired provider feedback. Questions addressed the following domains: 1) provider role and hospital service; 2 ) provider utilization of the consult service; 3) factors prompting providers to use the consult service; 4) provider opinions about the quality of the consult service and their level of satisfaction with it; and 5) impact of the consult service on provider behavior. The online survey using Qualtrics software consisted of 19 quantitative questions with either set choices or Likert-type scales. The set choice answers also included an option of "other" with ability to describe. In addition, respondents were given space to list any factors that contributed to their ratings of the service quality and their satisfaction with the service.

\section{Survey implementation}

For the July 2012 to June 2013 time frame, a report generated from the electronic health record showed that 285 unique medical providers in our system had placed a consult order during that time. Of these, 20 had either left the institution or had invalid email addresses. Thus, a total of 265 medical providers comprised of attending physicians, residents, hospitalists (physicians who work exclusively in a hospital), nurse practitioners, and physician assistants received an email invite to participate in the survey. The survey software was set to automatically send up to three email reminders to providers who had not yet completed the survey. Participation was voluntary and anonymous, with a chance to win one of three $\$ 50$ gift cards after survey completion as an incentive. A total of 118 providers completed the survey (44.5\% response rate).

\section{Data collection and analysis}

Qualtrics software was used to collect the data and analyze frequency distributions. For open-ended questions, answers were coded and reported as quantitative data. Differences in provider behavior were examined in SAS 9.4 (SAS Institute Inc., Cary, NC, USA) using chi-square tests with two-tailed significance at $p<0.05$. The University of North Carolina's Non-Biomedical Institutional Review Board deemed this study exempt based on category 2 classification (i.e., survey, interview, public observation). 


\section{Results \\ Demographics}

Resident physicians represented the majority of the respondents (67\%), followed by attending physicians (18\%). Twothirds of the responding providers reported spending at least 26 weeks on an inpatient service during the study period. Providers who responded to the survey represented the following clinical departments: general internal medicine (33\%), surgery (19\%), family medicine $(17 \%)$, neurology $(10 \%)$, psychiatry (9\%), and other hospital services (12\%). These percentages mirror the survey sample, with the exception of surgery, where fewer providers responded to the survey.

\section{Utilization of the consult service}

A majority of the respondents reported that they either "often" or "sometimes" put in a tobacco cessation consult order (35\% and $36 \%$, respectively), with $18 \%$ stating that they put in a consult "most times" and only $12 \%$ reporting "rarely" putting in a consult. Almost all (90\%) reported that it was "very easy" or "easy" to place a consult order.

Two factors were endorsed by over $60 \%$ of providers as influencing their decision to order a consult: 1) patient interest in quitting smoking and 2) belief based on previous experience that the consult service benefits patients (Figure 1).

\section{Impact on behavior}

We asked providers to indicate how exposure to or utilization of the tobacco consult service had influenced their counseling and prescribing behaviors. The majority of providers indicated greater likelihood of discussing tobacco use, offering cessation medications in the hospital, prescribing cessation medications at discharge, and including the recommendations in discharge summaries (Figure 2). We then examined how the likelihood of engaging in tobacco treatment interventions differed between providers who ordered a consult "sometimes or rarely" and providers who ordered a consult "often or most times". Those who ordered a consult "often" or "most times" were significantly more likely to provide tobacco cessation medication to inpatients, prescribe tobacco cessation medication at discharge, and address a patient's tobacco use at the first post-discharge clinic visit, as a result of exposure to or utilization of the consult service (Table 1).

Among providers who ordered consults "most of the time", internal medicine and family medicine providers stated that experience with the consult service made them more likely to prescribe cessation medication at discharge (77\%) than providers in specialized fields $(60 \% ; p=0.05)$. Providers who received at least one form of communication from the consult team (i.e., page, electronic medical record phone message, face to face, other; $74 \%$ ) were also more likely to prescribe cessation medication at discharge, compared to providers who received no communication from the consult team $(33 \% ; p<0.001)$.

\section{Importance, quality, and satisfaction}

Nearly all providers (97\%) endorsed the importance of the inpatient tobacco treatment service. A majority of the

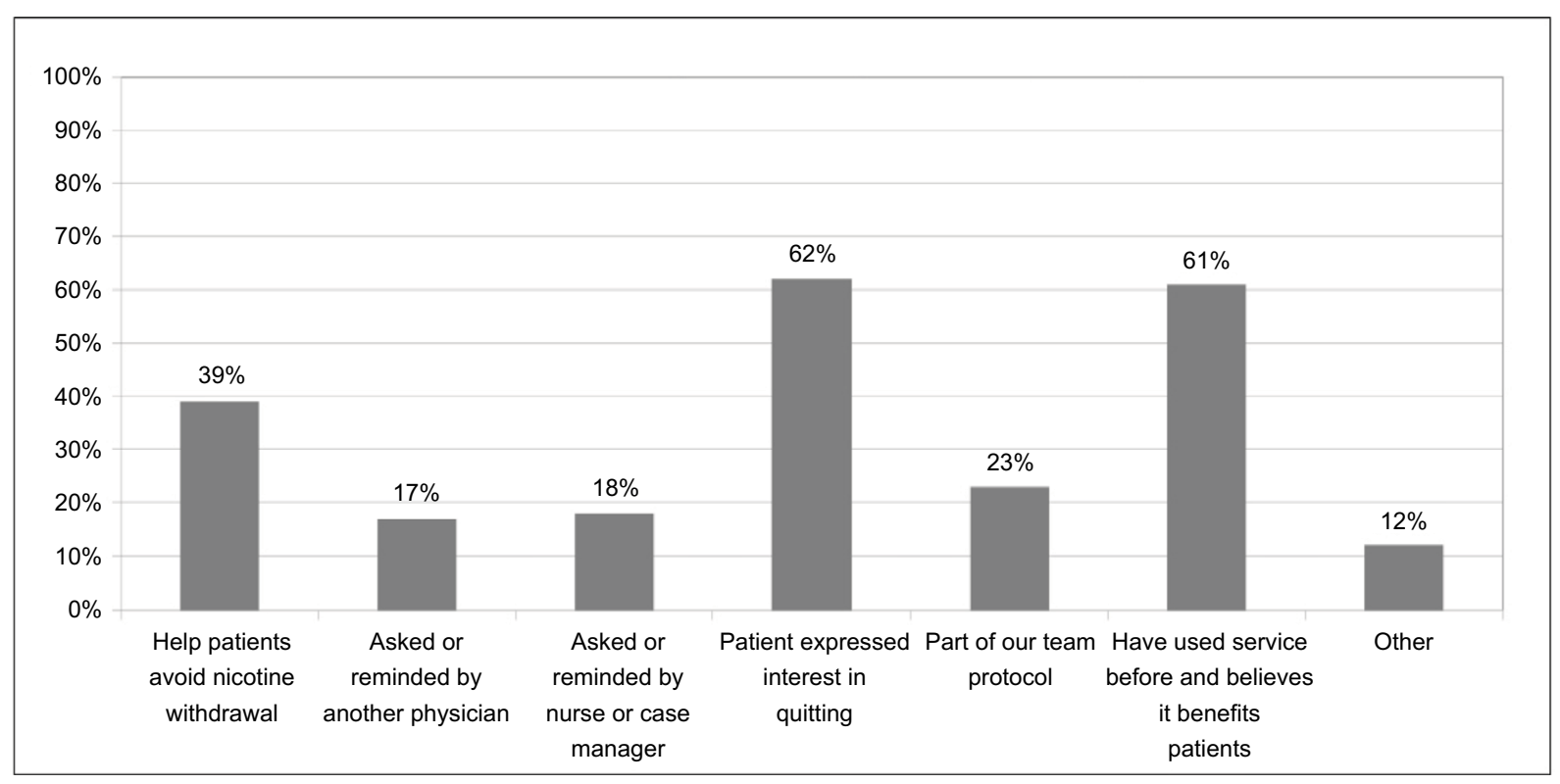

Figure I Factors positively impacting consults $(\mathrm{N}=|\mathrm{I}| 8)$. 


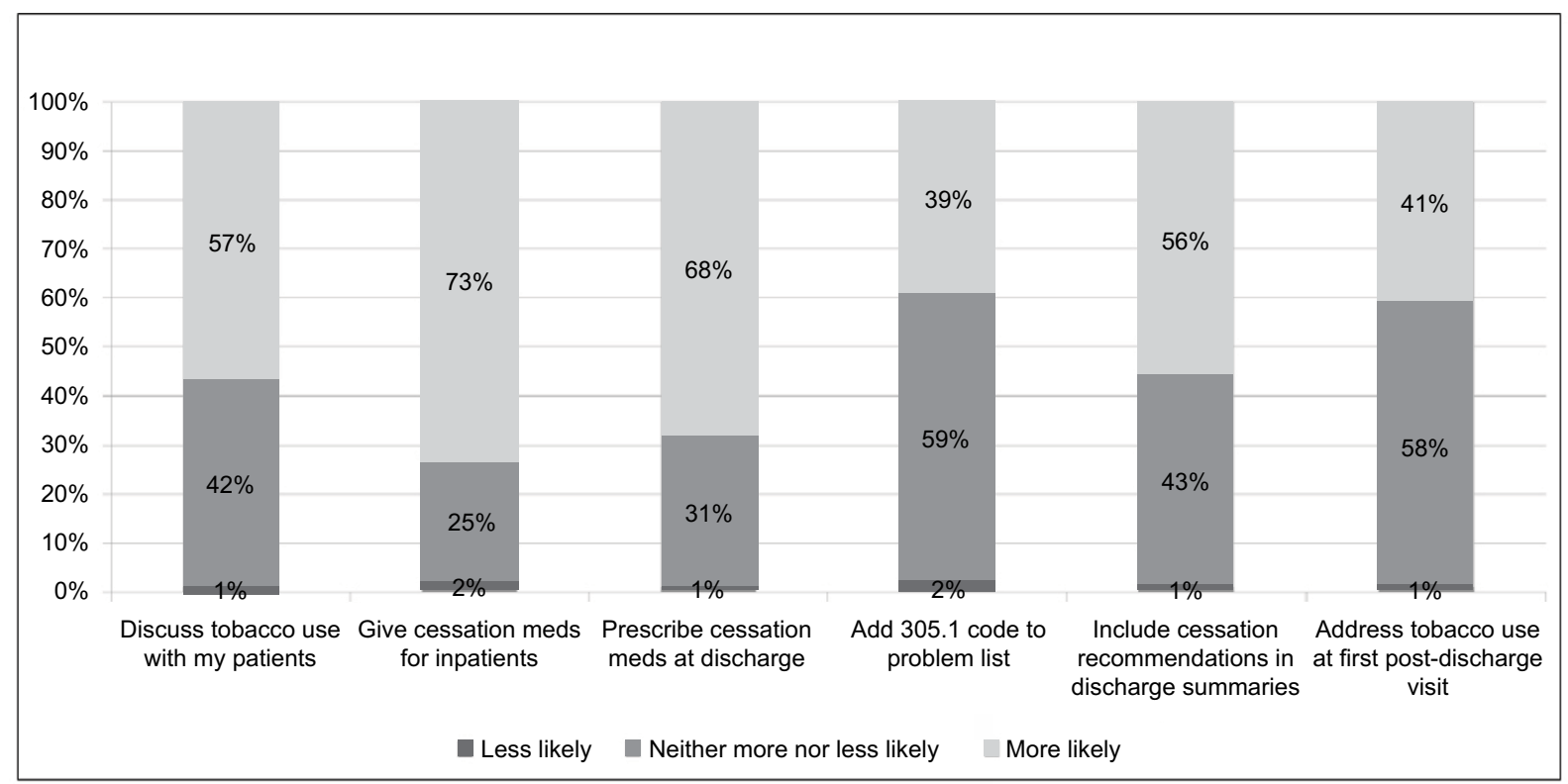

Figure 2 Impact of consult service on provider counseling behavior $(\mathrm{N}=\mid \mathrm{I} 8)$.

Abbreviation: meds, medications.

Table I Provider counseling and prescribing behavior, by frequency of ordering a tobacco treatment consult $(\mathrm{N}=\mathrm{I} / 8)$

\begin{tabular}{|c|c|c|c|}
\hline Outcome & $\begin{array}{l}\text { Providers ordering } \\
\text { consult "sometimes or } \\
\text { rarely", \% }\end{array}$ & $\begin{array}{l}\text { Providers ordering } \\
\text { consult "often or } \\
\text { most" times, \% }\end{array}$ & p-value \\
\hline More likely to discuss tobacco use with inpatients & 49 & 63 & 0.13 \\
\hline More likely to provide tobacco cessation medication to inpatients & 61 & 82 & 0.01 \\
\hline More likely to prescribe tobacco cessation medication at discharge & 58 & 77 & 0.02 \\
\hline More likely to add tobacco use disorder to the problem list & 34 & 45 & 0.20 \\
\hline More likely to include tobacco cessation recommendations in discharge summaries & 49 & 64 & 0.10 \\
\hline More likely to address patient's tobacco use at first post-discharge clinic visit & 31 & 51 & 0.03 \\
\hline
\end{tabular}

providers rated the tobacco treatment service as "excellent" $(59 \%)$. Reasons given included ease of ordering, promptness of responding to consult, expertise of consultants, and consult team recommendations. The great majority (88\%) reported being "satisfied" or "very satisfied" with the tobacco treatment service. When asked for the basis of their rating, over $80 \%$ of respondents listed easy access to the consult service and recommendations for appropriate tobacco cessation medication (Figure 3).

Given the multiple means of communication available between the consult team and providers, we asked the providers how they preferred to receive an update on completed consults. Providers could choose more than one form of communication. Most (70\%) preferred a message through the electronic health record, $42 \%$ preferred a page, and $21 \%$ indicated preference for face-to-face communication. Providers who received at least one form of communication (e.g., page, phone, face, other) from the consult team were more likely to rate their overall satisfaction with the consult service as "very satisfied", compared to providers not receiving any communication from the team $(47 \%$ and $22 \%$, respectively, $p=0.05)$.

\section{Discussion}

Hospitalization offers a critical teachable moment for tobacco cessation, and the importance of addressing tobacco use as part of hospital care is increasingly recognized. ${ }^{11}$ Effectively treating tobacco use in the hospital setting requires system changes to increase identification, counseling, and follow-up with patients who use tobacco. ${ }^{7,19}$ This research shows that having a tobacco treatment consult service available in the hospital setting appears to be a valued service by the providers; moreover, the service impacts providers' behaviors in both addressing tobacco use with patients and providing tobacco treatment medication and counseling. By impacting provider behavioral change, the service can lead to a more sustainable cultural change for addressing tobacco with hospitalized patients, particularly those who may have less 


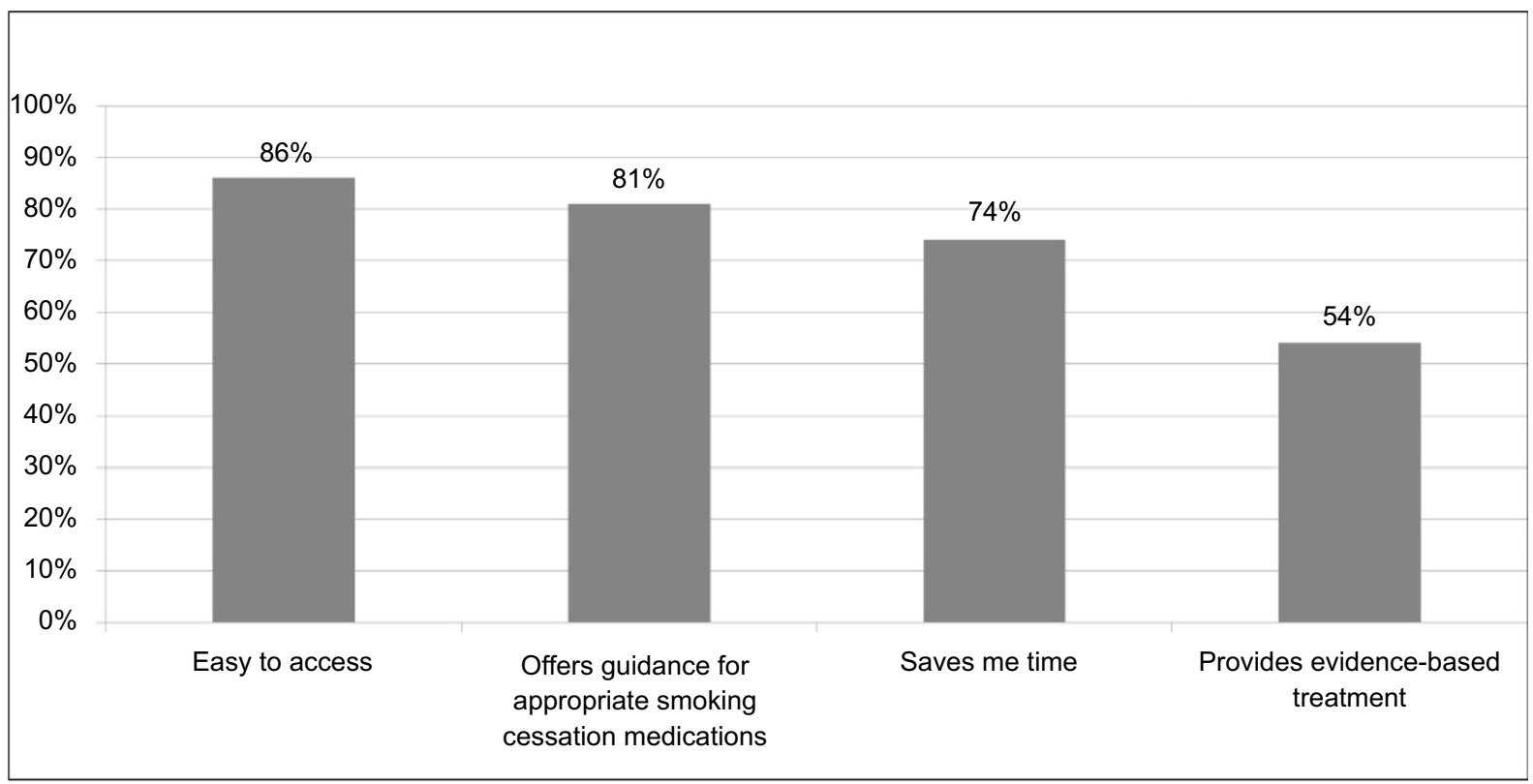

Figure 3 Most valuable characteristics of the consult service $(\mathrm{N}=\mid \mathrm{I} 8)$.

access to tobacco treatment services. Based on the survey, in addition to having a greater impact on the tobacco treatment of the current patients, there is an increased likelihood that future patients will also benefit from the provider addressing tobacco usage.

The goal of developing the inpatient tobacco treatment counseling program was to not only offer bedside counseling to inpatients who used tobacco, but also to provide education to the medical providers on how to best assist their patients in becoming tobacco free. Ultimately, provider satisfaction with a consult service increases the provider's usage of the service, has a greater likelihood to lead to culture change, and assists with the sustainability of the service. Culture change, achieved through educating providers about the benefits of the service and updating treatment protocols to reflect best practices, can increase the sustainability and impact of the program. ${ }^{17-20}$ Change in protocol, in this case placing orders for tobacco treatment consults for hospitalized patients, can positively impact the quantity and quality of programs and services. ${ }^{20}$

The survey results indicate that a high percentage of the ordering providers were satisfied with the tobacco treatment consults. Obtaining feedback from the providers on the positive and negative aspects of the service helps to know how to tailor the service to be most beneficial. Since providers state that they do not have time to thoroughly address tobacco use and that they value knowing there are resources to extend their care, this study confirms that patients are more likely to have their providers address tobacco use and prescribe appropriate medications when a dedicated treatment service exists to collaborate and assist providers.

Multidisciplinary teams are an integral part of the hospital structure. Support and buy-in from hospital providers is a key aspect for program success and sustainability. The inpatient tobacco cessation consult service is widely utilized and highly valued as an extension of provider care. As a consult service, communication with the consulting providers is the key to patient care and to optimally address inpatient tobacco use. Survey results also indicated that providers valued communication with the consult team, as there was a direct correlation between communication and increased provider satisfaction. Consult service promotion took place through educational in-services and communication directly from the tobacco treatment staff to the providers. While this method helped to increase the visibility and knowledge about the program, the majority of the providers stated that they were prompted to put in the consult by someone else. This emphasizes that additional educational sessions are helpful and that identifying key champions on different inpatient services could be beneficial in ongoing education of staff about the service. The role of nursing and case managers in reminding providers to place consults, as affirmed by the survey participants, offers another avenue for engaging hospital staff to support patients in receiving evidence-based treatment for tobacco use during their hospital stay. 


\section{Limitations}

We attempted to survey all providers who had ordered a tobacco treatment consult; however, frequent staff turnover in a teaching hospital meant we could only access those providers who continued to work in our system. Some resident physicians had graduated and left the institution and, therefore, could not be contacted. Developing an efficient system to educate new staff about how to use the service would likely increase utilization and collaboration. In cases where a nurse placed a consult in the doctor's name, the provider may not have recalled placing a consult, and thus declined to participate. We did not include nursing staff, since we had no accurate way to identify those who had placed these consults. Furthermore, we did not collect data from providers who had not utilized the tobacco treatment consult service. Moreover, this study also relied on provider self-report regarding current practices; some studies suggest self-report may not align with actual behavior. ${ }^{6}$

\section{Directions for future research}

Advances in electronic health record implementation and other health information technology systems enable greater consistency in documentation of measures of health, including tobacco use status. ${ }^{21}$ Research suggests that computer-based alerts improve provider likelihood of adhering to practice guidelines. ${ }^{22,23}$ While the consult service continues to grow, both in numbers of providers ordering consults and number of patients for whom orders are submitted, there are ways that the electronic medical record system could be used to help capture even more of the patients who use tobacco. Additionally, these data can be used to encourage other hospitals to adopt a similar program, especially as it aligns with the Joint Commission tobacco use treatment core measures set. ${ }^{12,24}$

Expanding the survey to include other members of the interdisciplinary team, such as nurses and clinical case managers who also order tobacco treatment consults, and further analysis of which services and/or medical issues lead to the most and fewest consult referrals could provide direction for improving the quality and effectiveness of the service. Additionally, gaining feedback from providers who do not use the consult service would be helpful in understanding the barriers to placing consult orders. Understanding why some providers are not utilizing the service would allow the program to better target changes, such as in education about the service or how to place consults. Finally, future research that compares provider knowledge and practice around inpatient tobacco use treatment in hospitals with and without a consult service would be needed to make a clearer causal link related to the service's impact on providers' knowledge and practice.

\section{Acknowledgments}

The authors thank the many hospital staff and providers who support this program, Mrudu Herbert, MD, MPH for her assistance with literature review, and Sara Harwood, MSW, MPH for her assistance with the development of the provider survey.

This work was an unfunded project of the UNC NDP. The UNC NDP programs are funded by the UNC Department of Family Medicine, UNC Health Care, and the Lineberger Comprehensive Cancer Center. A 2-year Pfizer grant helped fund the initial 2 years of NDP's inpatient consult team.

\section{Disclosure}

The authors report no conflicts of interest in this work.

\section{References}

1. Fiore M, Jaen C, Baker T, et al. Treating Tobacco Use and Dependence: 2008 Update: Clinical Practice Guideline. Rockville, MD: US Department of Health and Human Services; 2008.

2. Fellows JL, Mularski R, Waiwaiole L, et al. Health and economic effects from linking bedside and outpatient tobacco cessation services for hospitalized smokers in two large hospitals: study protocol for a randomized controlled trial. Trials. 2012;13(1):129.

3. Faseru B, Turner M, Casey G, et al. Evaluation of a hospital-based tobacco treatment service: outcomes and lessons learned. J Hosp Med. 2011;6(4):211-218.

4. Gadomski AM, Gavett J, Krupa N, Tallman N, Jenkins P. Effectiveness of an inpatient smoking cessation program. J Hosp Med. 2011;6(1):E1-E8.

5. Chu ES, Hakkarinen D, Evig C, et al. Underutilized time for health education of hospitalized patients. J Hosp Med. 2008;3(3):238-246.

6. Conroy MB, Majchrzak NE, Silverman CB, et al. Measuring provider adherence to tobacco treatment guidelines: a comparison of electronic medical record review, patient survey, and provider survey. Nicotine Tob Res. 2005; 7(Suppl 1):S35-S43.

7. Gadomski AM, Stayton M, Krupa N, Jenkins P. Implementing a smokefree medical campus: impact on inpatient and employee outcomes. J Hosp Med. 2010;5(1):51-54.

8. Rigotti NA, Arnsten JH, McKool KM, Wood-Reid KM, Pasternak RC, Singer DE. Smoking by patients in a smoke-free hospital: prevalence, predictors, and implications. Prev Med. 2000;31(2 Pt 1):159-166.

9. Stevens VJ, Glasgow RE, Hollis JF, Mount K. Implementation and effectiveness of a brief smoking-cessation intervention for hospital patients. Med Care. 2000;38(5):451-459.

10. Kim SH, Lee JA, Kim KU, Cho HJ. Results of an inpatient smoking cessation program: 3-month cessation rate and predictors of success. Korean J Fam Med. 2015;36(2):50-59.

11. Rigotti N, Munafo MR, Stead LF. Interventions for smoking cessation in hospitalised patients. Cochrane Libr. 2007. Available from: http:// onlinelibrary.wiley.com/doi/10.1002/14651858.CD001837.pub2/full. Accessed February 11, 2015.

12. The Joint Commission. Specifications Manual for National Hospital Inpatient Quality Measures. Tobacco Treatment. Available from: https:// www.jointcommission.org/specifications_manual_for_national_hospital_inpatient_quality_measures.aspx. Accessed September 13, 2017.

13. Nahhas GJ, Cummings KM, Talbot V, Carpenter MJ, Toll BA, Warren GW. Who opted out of an opt-out smoking-cessation programme for hospitalised patients? J Smok Cessat. 2016:1-6. 
14. Slattery C, Freund M, Gillham K, et al. Increasing smoking cessation care across a network of hospitals: an implementation study. Implement Sci. 2016;11(1):28.

15. Reid RD, Mullen KA, Slovinec D'Angelo ME, et al. Smoking cessation for hospitalized smokers: an evaluation of the "Ottawa Model." Nicotine Tob Res. 2009;12(1):11-18.

16. Liu SK, Prior E, Warren C, Brown T, Snide J, Butterly JR. Improving the quality of care for the hospitalized tobacco user one institution's transformational journey. J Cancer Educ. 2010;25(3):297-301.

17. Melnyk BM. Building cultures and environments that facilitate clinician behavior change to evidence-based practice: what works? Worldviews Evid Based Nurs. 2014;11(2):79-80.

18. Kotter JP. Leading change: why transformation efforts fail [1995]. Available from: http://www.gsbcolorado.org/uploads/general/PreSessionReadingLeadingChange-John_Kotter.pdf. Accessed March 1, 2017.

19. Wiltsey Stirman S, Kimberly J, Cook N, Calloway A, Castro F, Charns M. The sustainability of new programs and innovations: a review of the empirical literature and recommendations for future research. Implement Sci. 2012;7(1):17.
20. Greenhalgh T, Robert G, Bate SP, Kyriakidou O, MacFarlane F. How to spread good ideas: A systematic review of the literature on diffusion, spread and sustainability of innovations in health service delivery and organisation. London: NHS SDO Programme; 2004. Available from: http://discovery.ucl.ac.uk/180101/. Accessed March 1, 2017.

21. Moody-Thomas S, Nasuti L, Yi Y, Celestin MD, Horswell R, Land TG. Effect of systems change and use of electronic health records on quit rates among tobacco users in a public hospital system. Am J Public Health. 2015;105(Suppl 2):e1-e7.

22. Magnus M, Herwehe J, Andrews L, et al. Evaluating health information technology: provider satisfaction with an HIV-specific, electronic clinical management and reporting system. AIDS Patient Care STDs. 2009;23(2):85-91.

23. Wu CY, Chang CK, Robson D, et al. Evaluation of smoking status identification using electronic health records and open-text information in a large mental health case register. PLoS One. 2013;8(9):e74262.

24. Fiore MC, Goplerud E, Schroeder SA. The joint commission's new tobacco-cessation measures will hospitals do the right thing? $N$ Engl J Med. 2012;366(13):1172-1174.
International Journal of General Medicine

\section{Publish your work in this journal}

The International Journal of General Medicine is an international, peer-reviewed open-access journal that focuses on general and internal medicine, pathogenesis, epidemiology, diagnosis, monitoring and treatment protocols. The journal is characterized by the rapid reporting of reviews, original research and clinical studies across all disease areas.

\section{Dovepress}

The manuscript management system is completely online and includes a very quick and fair peer-review system, which is all easy to use. Visit http://www.dovepress.com/testimonials.php to read real quotes from published authors.

Submit your manuscript here: https://www.dovepress.com/international-journal-of-general-medicine-journal 